\title{
DISCRETE COSMOLOGY APPLICATION CONCERNING DARK MATTER HALOS
}

\author{
R.D. Brilenkov \\ Odessa I.I.Mechnikov National University, \\ Dvoryanskaya st. 2, Odessa 65082, Ukraine, \\ ruslan.brilenkov@gmail.com
}

\begin{abstract}
We applied discrete cosmology for investigation of the density profiles of dark matter (DM) halos of clusters of galaxies. Comparing the derived velocity dispersion with the experimental data for the Coma cluster, we found the effective radius for the mass distribution inside this galaxy cluster. Our estimates give an opportunity to restrict the parameters of the Navarro-Frenk-White profile for considered cluster.
\end{abstract}

Keywords: dark matter, clusters of galaxies.

It is generally known, our Universe is dark: dark energy and dark matter contribute approximately $69 \%$ and $26 \%$ into total mass-energy balance in the Universe, respectively.

We found potentials, which satisfy the Poisson equation and give us an opportunity to consider motion of test massive bodies and light taking into account gravitational attraction to inhomogeneities inside the galaxies, groups and clusters of galaxies and cosmological expansion of the Universe:

$$
\triangle \Phi=\frac{1}{R} \frac{d^{2}}{d R^{2}}(R \Phi)=4 \pi G_{N} \rho_{\mathrm{ph}},
$$

where $G_{N}$ is the Newtonian gravitational constant and $\rho_{\mathrm{ph}}$ is the physical rest mass density of the bound system.

After that we introduce the distance radius of zero acceleration surface (at which the gravitational attraction and cosmological expansion compensate each other):
Using the NFW density profile of DM halo of clusters of galaxies as the most commonly used profile:

$$
\rho_{\mathrm{ph}}(R)=\frac{4 \rho_{s}}{\frac{R}{R_{s}}\left(1+\frac{R}{R_{s}}\right)^{2}}, \quad R_{s}=\mathrm{const}, \quad \rho_{s}=\rho\left(R_{s}\right)
$$

we obtain, with the help of the observable data, the preferable profile parameters for the Coma cluster: $R_{200} \approx 1.77 h^{-1} \mathrm{Mpc}=2.61 \mathrm{Mpc}$ and the concentration parameter $c=3 \div 4$

The most galaxies are concentrated inside a sphere of effective radius $R_{\text {eff }} \sim 3.7 \mathrm{Mpc}$ for the Coma cluster (Abell 1656).

We also found line-of-sight velocity dispersion $1004 \mathrm{~km} \mathrm{~s}^{-1}$. The observations give very close value $1008 \mathrm{~km} \mathrm{~s}^{-1}$ for this cluster.

Acknowledgements. I would like to thank my supervisors Alexander Zhuk and Maxim Eingorn who helped me to get these results.

\section{References}

Eingorn M., Zhuk A.: 2012, Hubble flows and gravitational potentials in observable Universe, JCAP, 09, 026; arXiv:1205.2384.

Eingorn M., Kudinova A., Zhuk A.: 2013, Dynamics of astrophysical objects against the cosmological background, JCAP, 04, 010; arXiv:astro-ph/1211.4045.

Eingorn M., Zhuk A.: 2014, Remarks on mechanical approach to observable Universe, JCAP, 05, 024; arXiv:astro-ph/1309.4924.

$$
R_{H}=\left[\frac{G_{N} M}{\ddot{a} / a}\right]^{1 / 3}
$$

